\title{
Evolution of Neutral Gas in Galaxies over Cosmic Time with SKA pathfinder instruments.
}

\author{
K.J. van der Heyden *, A. Bouchard, B.W. Holwerda, S.-L. Blyth \& W.J.G. de Blok, \\ Department of Astronomy, University of Cape Town, Private Bag X3, Rondebosch 7701, \\ Republic of South Africa \\ E-mail: heydendast.uct.ac.za
}

\begin{abstract}
Exactly how the cosmic abundance of neutral hydrogen (H I) evolves with redshift is currently unknown. This is a challenging problem for constraining models of galaxy formation. We investigate the possibilities of measuring the $\mathrm{H}$ I mass function (H IMF) of galaxies as a function of redshift for the next generation of all-sky surveys. We use a simple non-evolving H IMF to predict the number counts of sources likely to be detected by H I surveys on next generation radio telescopes, particularly the South African SKA pathfinder telescope MeerKAT. These results show that future H I surveys leading up to the advent of the SKA era will provide important and powerful tests of theoretical models of galaxy formation.
\end{abstract}

Panoramic Radio Astronomy: Wide-field 1-2 GHz research on galaxy evolution - PRA2009

June 02 - 052009

Groningen, the Netherlands

${ }^{*}$ Speaker. 


\section{Introduction}

One of the motivations for building the Square Kilometre Array (SKA) is to study the evolving neutral gas content of galaxies throughout cosmic time. The distribution and kinematics of the $21 \mathrm{~cm}$ emission line of neutral hydrogen (H I) provide valuable insights into galaxy formation and evolution. An extragalactic survey of the entire sky contains information on the distribution of galaxies, the H I mass function, the dynamics of groups and the frequency of dwarf galaxies. Thanks to surveys such as HIPASS (H I Parkes All-Sky Survey; see Meyer et al. 2004) and more recently ALFALFA (Arecibo Legacy Fast ALFA Survey; see Giovanelli et al. 2005), we have a good understanding of the H I properties of galaxies in the low redshift Universe, at $\mathrm{z}<0.05$. In contrast, we know comparatively little about $\mathrm{HI}$ in galaxies at higher redshifts (i.e. $\mathrm{z}>0.05$ ). This is because detecting $\mathrm{H}$ I emission from individual galaxies requires an unreasonably large amount of observing time with current facilities. The evolution of $\mathrm{H} \mathrm{I}$ gas as a function of time is therefore currently poorly constrained but is vital for understanding the gas evolution the Universe. There are some estimates of $\mathrm{H}$ I at high redshifts ( $\mathrm{z}>1.5)$, but these have been deduced from QSO absorptionline systems (e.g. Péroux et al. 2003; Prochaska et al. 2005; Rao et al. 2006).

Our knowledge of the $\mathrm{H}$ I content between $\mathrm{z}=0.05-1.5$ is expected to dramatically improve over the next decade with the emergence of a series of next generation radio telescopes, culminating in the SKA. The SKA is expected to have the sensitivity to detect $\mathrm{H}$ I in galaxies out to redshifts $\mathrm{z} \sim 3$ (Blake et al. 2004; Braun 2007). A variety of SKA path-finders such as ASKAP, MeerKAT, APERTIF and EVLA will, however, be available on a much shorter timescale. These instruments will carry out H I surveys that, in some cases, will probe the properties of galaxies out to $\mathrm{z} \lessgtr 0.5$. The primary aim of this contribution is to explore the possibilities of constraining the H I mass function out to redshifts $\mathrm{z}>0.05$.

\section{Surveys}

Table 1 contains information on the two of the main SKA precursors as was based on information provided by de Blok (2009) \& Meyer (2009). This was used to predict the galaxy number counts for various survey parameters. The scientific assumptions are based on a HIMF as determined in Zwaan et al. 2005, based on data from the deepest systematic HI survey HIPASS $(\mathrm{N}=4315)$ to date. Their Schechter function parameters are $\alpha=-1.37$ for the faint end slope, $\log \left(M_{\mathrm{HI}}^{*}\right)=9.80 \mathrm{M}_{\odot}$ the characteristic HI mass, and $\Omega *=6.0 \cdot 10^{-3} \mathrm{Mpc}^{-3} \mathrm{dex}^{-1}$. Their results were found to be in good agreement with the HIPASS Bright Galaxy Catalogue, a complete but smaller, sub-sample of galaxies (Zwaan et al. 2003). We adopt the same cosmological parameters of $\mathrm{H}_{0}=75 \mathrm{Mpc} \mathrm{km}^{-1} \mathrm{~s}^{-1}, \Omega_{m}=0.3$, and $\Omega_{\Lambda}=0.7$. We assume a non-evolving $\mathrm{H}$ IMF and constant $\mathrm{rms}$ and beam size with redshift/frequency. We demand $\mathrm{S} / \mathrm{N}=5$ for robust detection, and assume a channel width of $25 \mathrm{~km} / \mathrm{s}$. We also assume that we are able to optimally smooth the data. We also investigate the predicted counts for similar ASKAP surveys.

\section{Results \& Discussion}

Figs. 1-3 illustrate the results based on these simulations are through a variety of plots in. The plots displayed Fig. 1 compares the number $(\mathrm{N})$ of galaxy detections as for increasing redshift $z$ 


\begin{tabular}{ccc}
\hline Telescope Parameters & MeerKAT & ASKAP \\
\hline FOV $\left(\mathrm{deg}^{2}\right)$ & 0.8 & 30 \\
\# Dishes & 80 & 36 \\
Aperture efficiency & 0.7 & 0.7 \\
$\mathrm{~T}_{\text {sys }}$ & 30 & 50 \\
$\mathrm{rms}(\mathrm{mJy})(8 \mathrm{hrs}, 25 \mathrm{~km} / \mathrm{s})$ & 0.6 & 1.4 \\
\hline
\end{tabular}

Table 1: Assumed telescope specifications.
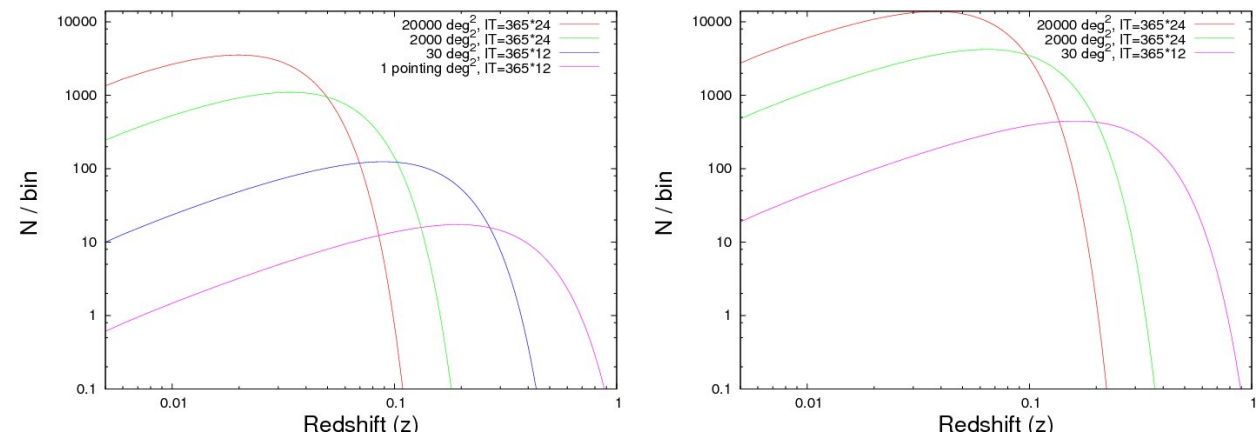

Figure 1: Detected numbers of galaxies $(\mathrm{N})$ for increasing redshift $z$ in intervals of $\Delta z=0.002$ for a survey duration of $1 \mathrm{yr}$. The left plot corresponds to a MeerKAT observations, while the plot on the right corresponds to ASKAP observations. The different curves correspond to the survey areas indicated at the top of the plots.
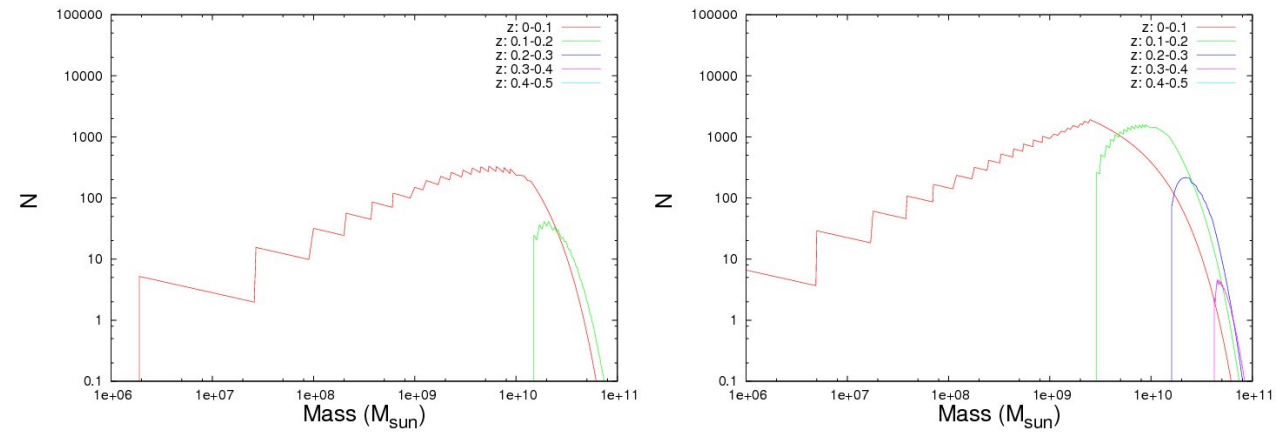

Figure 2: Detected numbers of galaxies $(N)$ for increasing $\mathrm{H}$ I mass in intervals of $\log \left(\mathrm{M}_{\mathrm{HI}}\right)=0.1 \mathrm{dex}$ for a survey duration of $1 \mathrm{yr}$. The left plot corresponds to a MeerKAT survey for a survey area of $2000 \mathrm{deg}^{2}$, while the right plot corresponds to the same survey performed with ASKAP. The different curves correspond to the red-shift intervals as indicated at the top of the plots. The wiggles in the curves arise from numerical issues in the calculation. 

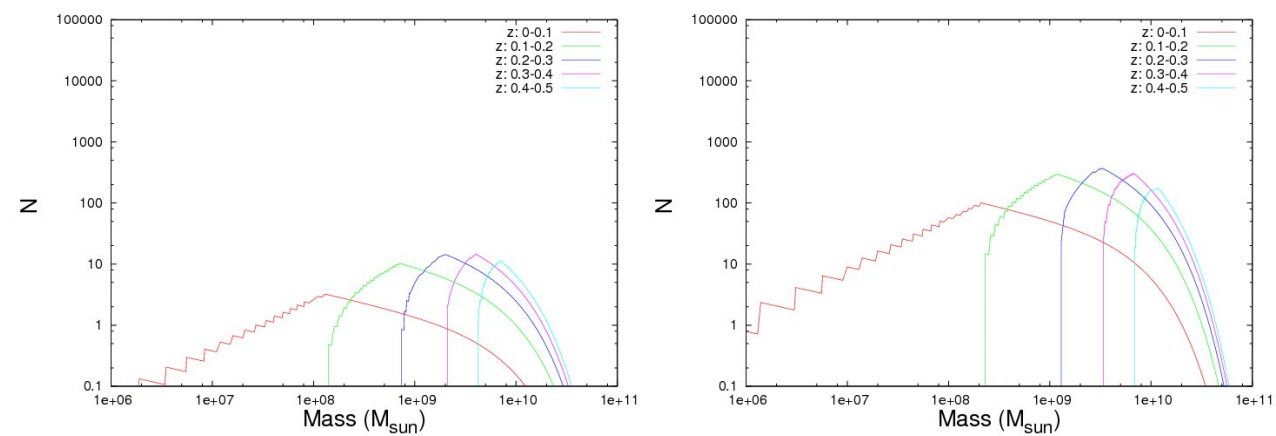

Figure 3: Similar to Fig. 2. The top left $\&$ right plots corresponds to MeerKAT surveys for a survey area of $2000 \& 0.8$ (i.e 1 pointing) $\mathrm{deg}^{2}$, respectively. The bottom left \& right plots corresponds to ASKAP surveys for a survey area of $2000 \& 30$ (i.e 1 pointing) $\mathrm{deg}^{2}$, respectively. The different curves correspond to the redshift intervals indicated at the top of the plots. The wiggles in the curves arise from numerical issues in the calculation.
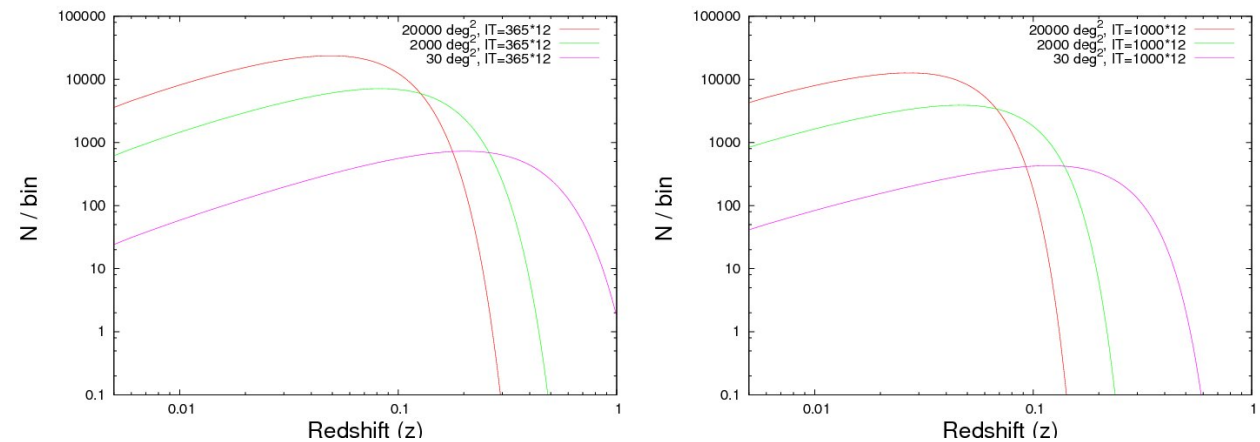

Figure 4: Detected numbers of galaxies $(\mathrm{N})$ increasing redshift $z$ in intervals of $\Delta z=0.002$ for a survey duration of 3 yrs with ASKAP. The plot on the left represents the optimally smoothed, while the plot on the right is for un-binned data.

for MeerKAT and ASKAP. The results for various survey areas (20000, 2000, $\left.30 \& 0.8 \mathrm{deg}^{2}\right)$ and a survey duration of 1 yr. Figs. 2-3 gives the number of detections as a function of galaxy mass (given in steps of $\log \left(\mathrm{M}_{\mathrm{HI}}\right)$ of $0.1 \mathrm{dex}$ ).

Achieving interesting $\mathrm{H}$ I galaxy sample sizes with pathfinder SKA surveys requires very substantial survey durations, of $\gtrsim 1$ yr. Good sampling $(\log (\mathrm{N}))$ down to below $\mathrm{M}_{\mathrm{HI}}^{*}$ can possibly be achieved out to $\mathrm{z} \lessgtr 0.2$ over $2000 \mathrm{deg}^{2}$ with ASKAP. It is clear from Figs. $1 \& 2$ that ASKAP will be a much better suited to large area H I surveys. This is because ASKAP has a much larger FOV than MeerKAT (i.e. 30 and $0.8 \mathrm{deg}^{2}$ respectively). MeerKAT, in contrast, will fare much better at pointed observations, where its superior sensitivity will allow one to probe the ill-constrained low mass end of the HIMF. This is illustrated in Fig. 3, which show the results for a single pointing observation over a period of $1 \mathrm{yr}$. MeerKAT is capable of detecting lower mass galaxies while ASKAP will detect many more high mass galaxies.

The results presented here (and generally throughout the literature) assumes that $5 \sigma$ detections yield robust results and also that we will be able to perform optimal smoothing. These assump- 


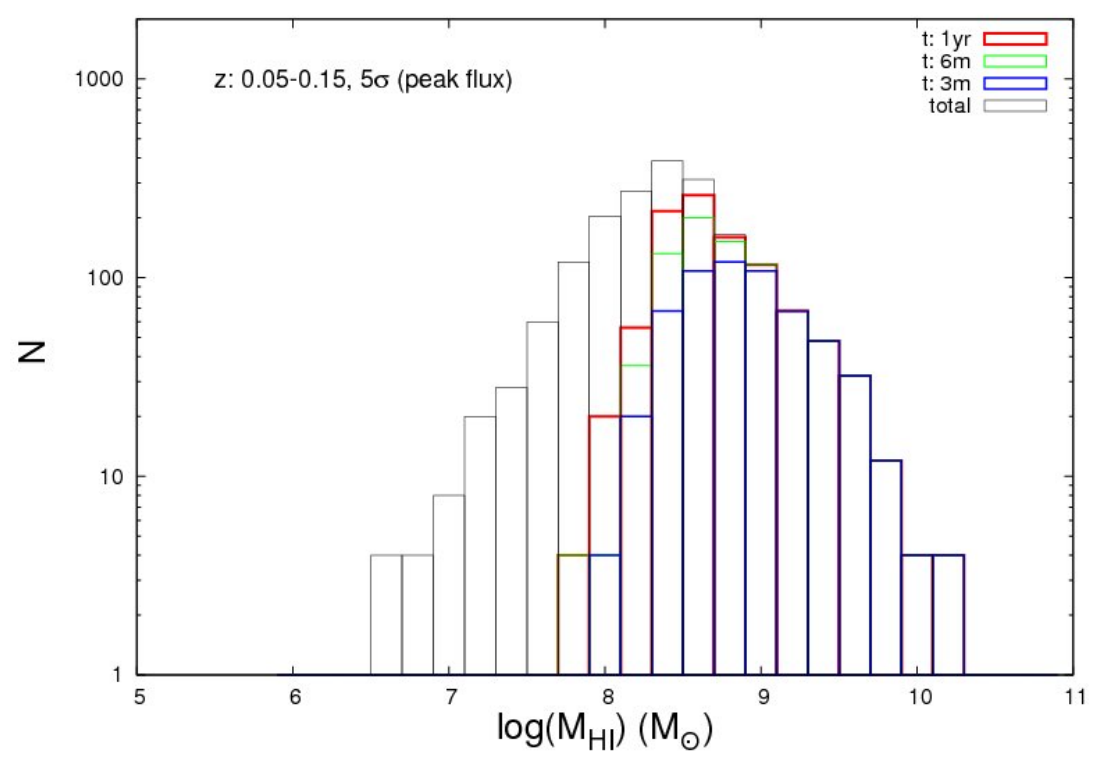

Figure 5: The numbers of galaxies $(\mathrm{N})$ as a function of their $\mathrm{H}$ I mass from the Obreschkow virtual sky catalogue in the redshift range $\mathrm{z}=0.05-0.15$. The the black histogram indicates the total counts. The coloured histograms are the detected counts corresponding to the integration times indicated at the top of the plots. The results are for 1 pointing with MeerKAT and assuming a $5 \sigma$ detection limit.

tions need still need to be vigorously tested. It is not clear whether, in practice, we will be able to perform optimal smoothing. The ability to perform optimal smoothing can lead to dramatically different results as illustrated in Fig. 4. Here the plot on the right show the the number of galaxy detections from a 3 yrs survey performed with ASKAP. The plot on the left show the results for the same survey but now assuming that optimal smoothing is possible. The ability to apply optimal smoothing allow for much higher detection rates and also for detections out to higher redshifts. Other issues like sample completeness, cosmic variance and source sizes also need to be considered. The effect of sample incompleteness is illustrated in Fig. 5. Here we see how many galaxies will go un-detected with a single pointing observation MeerKAT. The histograms in Fig. 5 are various observing times an a $5 \sigma$ detection threshold. The data are from the Obreschkow virtual sky catalogue (Obreschkow et al. 2009).

\section{Conclusion}

All four SKA path-finders are expected to be operational in the same timeframe. The very long survey durations and the different locations (APERTIF \& EVLA in the North and ASKAP \& MeerKAT in the South), survey speed and sensivity of the instruments indicates that complimentary surveys could then be carried out by the different facilities to maximize the total scientific return.

\section{References}

[1] Blake, C.A., Abdalla, F.B., Bridle, S.L. \& Rawlings, S., 2004, New Astronomy Review, 48, 1063 
[2] de Blok, W.J.G., 2009, this proceedings

[3] Braun, R., 2007, preprint [arXiv:astro-ph/0703746]

[4] Giovanelli, R., Haynes, M.P., Kent, B.R., et al., 2005, AJ, 130, 2598

[5] Meyer, M.J., 2009, this proceedings

[6] Meyer, M.J., Zwaan, M.A., Webster, R., et al., 2004, MNRAS, 350, 1195

[7] Obreschkow, D., Klöckner, H.-R., Heywood, I., Levrier, L., \& Rawlings,S., 2009, ApJ, in press

[8] Péroux C., McMahon, R.G., Storrie-Lombardi, L.J. \& Irwin, M.J., 2003, MNRAS, 346, 1103

[9] Prochaska, J. X., Herbert-Fort, S. \& Wolfe, A.M., 2005, ApJ, 635, 123

[10] Zwaan, M.A., Meyer, M.J., Staveley-Smith, L., \& Webster, R.L. 2005, MNRAS, 359, L30-L34

[11] Zwaan, M., Staveley-Smith, L., Koribalski, B.S. et al. 2003, AJ, 125, 2842 